\title{
Foetal Medicine-Who is to Practise It?
}

During the past few decades there has been a steady and large reduction in mortality rates of infants and young children, due no doubt as much to improvements in sanitation, housing, diet, education, and affluence, as to purely medical advances. Has the foetus shared in these benefits? Despite what is often assumed to the contrary, he has done so to quite an impressive extent. Over the 33 years from I93I to 1964, for instance, the stillbirth rate in England and Wales fell by $59 \%$ (from 4I to I 7 per I,000 births), and first-week deaths by $46 \%$ (from 22 to 12 per I,000 births).

Nevertheless, further substantial improvements in foetal mortality and, equally important from its long-term effects, in foetal morbidity seem likely to require the development of new techniques which will allow a much more direct approach to the foetus than has been possible hitherto. Up to now the formidable inaccessibility of the human foetus has meant that foetal medicine (apart perhaps from foetal electrocardiography) has virtually not existed. In an age when Man has been able to measure most things from an atom to a galaxy, it is thus paradoxical that to measure his own size during the most critical and precarious period of his life, he still has to depend upon the extreme fallibility of the palpating hand.

The obstetrician's predominating interest has traditionally centred on the mother, while the paediatrician has had to wait on the touchline without access to the baby until the moment of birth, an arbitrarily timed event in the growth and development of an individual. In a sense, therefore, the foetus has been 'nobody's baby'.

These thoughts are prompted by the work of Saling, an article by whom, and a review of whose recent book, appear elsewhere in this issue. With the advent of the techniques of amnioscopy and foetal blood sampling developed by Saling, and of amniocentesis and foetal transfusion due to Bevis and to Liley, we witness the end of the long period of foetal inaccessibility and, we hopefully believe, the start of the science of foetal medicine.

The question which provides the title to this note is intentionally provocative. The foetal environment being provided by the mother, whoever cares for the foetus must needs be an obstetrician, yet an obstetrician who should be orientated equally towards both of the individuals in his charge. And having cared for the foetus for nine months, and seen it through the critical delivery phase, it would be only logical for the same person to continue without a break to carry it through the immediate neonatal phase.

Is a new kind of doctor needed, at least at the academic level, who, by combining the interests and skills of both the obstetrician and the paediatrician, can act as the foetus's doctor? 